\title{
BMJ Open Combination therapy of anabolic agents and bisphosphonates on bone mineral density in patients with osteoporosis: a meta-analysis of randomised controlled trials
}

\author{
Shenghan Lou, ${ }^{1,2}$ Houchen Lv, ${ }^{1}$ Zhirui Li, ${ }^{1}$ Licheng Zhang, ${ }^{1}$ Peifu Tang ${ }^{1}$
}

To cite: Lou S, Lv H, Li Z, et al. Combination therapy of anabolic agents and bisphosphonates on bone mineral density in patients with osteoporosis: a meta-analysis of randomised controlled trials. BMJ Open 2018;8:e015187. doi:10.1136/ bmjopen-2016-015187

- Prepublication history and additional material for this paper are available online. To view these files, please visit the journal online (http://dx.doi. org/10.1136/bmjopen-2016015187).

$\mathrm{SL}$ and $\mathrm{HL}$ contributed equally.

Received 16 November 2016 Revised 2 January 2018 Accepted 29 January 2018

\section{Check for updates}

${ }^{1}$ Department of Orthopedics, General Hospital of Chinese PLA, Beijing, China

2Department of Spine Surgery, First Affiliated Hospital of Harbin Medical University, Harbin, China

Correspondence to

Dr Peifu Tang;

pftang301@163.com

\section{ABSTRACT}

Objective We aimed to determine whether the concomitant combination therapy of anabolic agents and bisphosphonates produces more effects on bone mineral density (BMD) than anabolic agents alone in patients with osteoporosis.

Methods We searched MEDLINE, EMBASE and the Cochrane Library for publications from 1 January 1980 to 1 August 2016 to identify all the randomised controlled trials (RCTs) and quasi-RCTs. The primary outcome was the mean per cent changes in BMD at the lumbar spine, the total hip and the femoral neck with an optimal period of treatment (6 to 12 months). The secondary outcome was the mean per cent changes in BMD at the same sites with the full period of recommendation (18 to 24 months). $A$ random-effects model was used to estimate the standardised mean differences (SMDs) and the 95\% Cls. Results Seven studies, with 747 patients, were included. With the optimal period, the concomitant combination therapy demonstrated a significant advantage over a monotherapy in BMD improvement at the total hip (SMD $0.42 ; 95 \% \mathrm{Cl} 0.26$ to 0.58 ) and the femoral neck (SMD $0.30 ; 95 \% \mathrm{Cl} 0.14$ to 0.46 ), but not for the spine BMD (SMD $0.13 ; 95 \% \mathrm{Cl}-0.17$ to 0.43 ). With the full period, the concomitant combination therapy did not improve the BMD at the lumbar spine (SMD $-0.06 ; 95 \% \mathrm{Cl}-0.71$ to 0.59 ), the total hip (SMD $0.05 ; 95 \% \mathrm{Cl}-0.71$ to 0.82 ) and the femoral neck (SMD $-0.32 ; 95 \% \mathrm{Cl}-1.15$ to 0.50 ). Conclusions Compared with anabolic monotherapy, the concomitant combination therapy of anabolic agents and bisphosphonates significantly improved the BMD at the total hip and femoral neck with a shorter term (6 to 12 months) and produced similar benefits on BMD for the longer term (18 to 24 months). Also, the effect of concomitant combination therapy might be affected by the dose of anabolic agents.

PROSPERO registration number CRD42016041335.

\section{INTRODUCTION}

Osteoporosis is characterised by low bone mass and a microarchitectural deterioration of the bone tissue. ${ }^{1}$ Due to the high mortality and morbidity, osteoporosis-related fractures
Strengths and limitations of this study

- This meta-analysis is more rigorous than the previous meta-analyses, including trials, with more reliable results.

- The included trials were all randomised controlled trials, and the overall quality of the evidence was assessed using the Grading of Recommendations Assessment, Development and Evaluation.

- Only seven studies were eligible for inclusion in this meta-analysis.

- Due to the limited number of the eligible trials and that the sample size of each trial was fairly small, a direct assessment of the antifracture efficacy was not performed.

have become a formidable public health threat. ${ }^{2-4}$ In the USA alone, the cost of fractures related to osteoporosis in 2005 was US $\$ 16.9$ billion, and it is estimated that this figure would increase to US $\$ 25.3$ billion by $2025 .^{5}$

To date, a range of pharmacological interventions is available for the treatment of osteoporosis. Depending on their mechanism of action, antiosteoporosis medications can be classified into either antiresorptive agents or anabolic agents. Antiresorptive agents include bisphosphonates, hormone replacement therapy, raloxifene, denosumab and calcitonin. Anabolic agents include the full-length molecule parathyroid hormone (PTH 1-84) and teriparatide (PTH 1-34). Despite various drugs, there is no evidence that single use of any agent can restore skeletal integrity in most patients with established osteoporosis. ${ }^{6}$ Theoretically, the 'ideal' antiosteoporosis drug would increase the new bone formation and inhibit bone resorption. ${ }^{7}$ Given this absence of the 'ideal' therapy, the concomitant combination therapy of anabolic and antiresorptive 
agents has been proposed as alternative. The rationale for combining anabolic and antiresorptive agents is that if bone formation is stimulated by an anabolic agent while bone resorption is inhibited by an antiresorptive agent, the concomitant combination therapy might attain superior bone mass and strength effects compared with monotherapy. ${ }^{8}$

Since bisphosphonates are the most commonly used antiresorptive medications for the treatment of osteoporosis, ${ }^{9}$ the concomitant combination therapy of anabolic agents and bisphosphonates was initially thought to be a promising approach. However, results of the concomitant use of bisphosphonates and anabolic agents were mixed. ${ }^{10-19}$ The earlier trials did not confirm this positive effect, ${ }^{10} 111517-19$ but trials in recent years reported a positive effect of the combination therapy. ${ }^{12-1416}$

To date, it remains unclear (1) whether bisphosphonates in concomitant combination with anabolic agents could produce more benefits than anabolic monotherapy, (2) whether the dose and type of antiosteoporosis agents could affect the effect of concomitant combination therapy and (3) when the combination therapy should be used. Thus, a meta-analysis was necessary to summarise the current evidence. Two meta-analyses ${ }^{20} 21$ on the topic have been published, indicating that the combination therapy was not superior to monotherapy with anabolic agent alone,$^{20}$ and even reduced the ability of anabolic therapy to increase the bone mineral density (BMD) at the lumbar spine, femoral neck and total hip. ${ }^{21}$ However, it should be noted that some serious problems existed in these two studies, such as (1) missing some key trials,${ }^{202}$ which determined the combination therapy had a higher increase in BMD than the monotherapy ${ }^{13}{ }^{16}$; (2) using repeated data ${ }^{20}$; (3) including trials which did not meet their inclusion criteria. ${ }^{20} 21$ These problems greatly reduced the credibility of their results.

Therefore, this meta-analysis of randomised controlled trials (RCTs) aimed to determine whether the concomitant combination therapy of anabolic agents and bisphosphonates produces more effects on BMD than an anabolic agent alone in patients with osteoporosis.

\section{METHODS}

This meta-analysis protocol was registered on the International Prospective Register of Systematic Reviews (CRD42016041335), and was developed following the principles of the Preferred Reporting Items for Systematic Reviews and Meta-Analyses guidelines. ${ }^{22}$ This study was not a human or animal experiment, so no ethical approval was required.

\section{Study inclusion and exclusion criteria}

Studies included in this meta-analysis meet the following inclusion criteria: (1) study design: RCT with a duration of at least 6 months; (2) study subjects: patients diagnosed with osteoporosis; (3) study intervention: patients in the treatment group received a concomitant combination therapy of anabolic agents and bisphosphonates, whereas patients in the control group received monotherapy with anabolic agents; (4) outcome measure: the outcome measurement included the mean per cent increases in BMD (measured by dual-energy X-ray absorptiometry) of the lumbar spine, femoral neck and total hip. Owing to the potential higher risk of osteosarcoma, treatment of anabolic drugs was recommended less than 24 months (the full period). ${ }^{23}{ }^{24}$ Moreover, owing to the subsequent resistance to anabolic agents, it was suggested that to increase $\mathrm{BMD}$, anabolic agents might best be used for periods (the optimal period) of 6 to 12 months or less. ${ }^{25}$ Thus, the primary outcome of interest was the BMD changes from an optimal period (6 to 12 months). The secondary outcome was the BMD changes from the full period (18 to 24 months). Exclusion criteria included non-RCTs or studies published as abstracts, review articles, editorials and letters.

\section{Data sources and search}

We systematically searched MEDLINE, EMBASE and the Cochrane Library from 1 January 1980 until 1 August 2016, with no language restrictions. Additionally, relevant studies were obtained by scanning reference lists of articles identified in the initial searches, relevant meta-analyses and systematic reviews.

The literature search was performed in duplicate by two independent authors (SL, HL). Search strategies were developed using text words as well as medical subject headings associated with terms relevant to 'osteoporosis', 'teriparatide', 'parathyroid hormone', 'bisphosphonate' together with 'randomized control trial'. The full search strategies used in MEDLINE, EMBASE and Cochrane Library are provided in online supplementary file S1.

\section{Study selection}

Our search records were imported into ENDNOTE X7 reference management software, and two authors (SL, HL) independently reviewed the titles and abstracts of the literature searches. Trials that did not meet the eligibility criteria were excluded. After excluding the duplicated and obviously irrelevant articles, the remaining studies were further reviewed by full-text reading to ascertain whether they should be included by the same eligibility criteria. For duplicate articles or publications reporting the same data in multiple articles, only the study with the most complete data and the longest follow-up would be included. After completion, both authors met and reviewed their selections for agreement. Any disagreements were resolved by discussion or by seeking an independent third author (ZL).

\section{Data extraction}

A standard data extraction form was created using Microsoft Excel 2016 to collect data of interest. The major categories of variables to be coded were: (1) study characteristics; (2) participant characteristics; (3) type of intervention (type, dose, duration) and (4) outcome 
characteristics. Information was carefully extracted from all the eligible publications independently by two authors (SL, HL), and disagreements were resolved through discussion or by seeking an independent third author (ZL). If the original data were not available, we calculated the data through the available coefficients. For example, we computed the mean from median and SD for SE, IQR or $\mathrm{P}$ values, according to the methods described in the Cochrane Handbook.

\section{Risk of bias assessment}

Two authors (SL, HL) independently assessed the risk of bias using the Cochrane risk-of-bias tool. ${ }^{26}$ The Cochrane risk-of-bias tool assessed bias across the following seven domains: (1) random sequence generation; (2) allocation concealment; (3) blinding of participants and personnel; (4) blinding of outcome assessment; (5) incomplete outcome data; (6) selective reporting; (7) other bias. Each domain was determined as 'low risk', 'unclear risk' or 'high risk'. For the first four domains, if the trial clearly reported adequate methods, it was regarded as a low risk of bias. If the trial did not clearly report the methods, it was regarded as an unclear risk of bias. If the trial reported inadequate methods, it was regarded as a high risk of bias. For the incomplete outcome data, we considered $\geq 20 \%$ loss to follow-up to represent a high risk of bias. For the selective reporting, we assessed it by comparing each publication with its corresponding published protocol, when available. For other sources of bias, we considered major imbalances in key baseline characteristics represent a high risk of bias. Any disagreements were resolved through discussion, and sometimes with another reviewer (ZL) if necessary.

\section{Statistical analysis}

Both primary outcomes and secondary outcomes of this study were changes in BMD, and all of them were continuous data, so the effect sizes were reported as standardised mean differences (SMDs) and 95\% CIs, using the generic inverse variance (IV) methods .

The meta-analysis was performed using a random$\mathrm{e} \square$ ects model, which provided more conservative estimated effects. To assess the heterogeneity of the results from individual studies, Cochrane's $Q$ statistic, the $I^{2}$ statistic $\left(\mathrm{I}^{2}>50 \%\right.$ as a threshold indicates significant heterogeneity) and $\mathrm{P}$ values $(\mathrm{P}<0.10$ as a threshold indicates significant heterogeneity) were used. ${ }^{27}$ The preplanned subgroup analyses were performed to explore the sources of heterogeneity based on the different types of antiosteoporotic agents or the different dose of anabolic agents. The publication bias was assessed visually with a funnel plot. Additional sensitivity analyses for the primary outcomes were done to explore the heterogeneity by omitting specific trials from the overall analysis. The meta-analysis was analysed using the statistical software packages Review Manager (V.5.3) and Comprehensive Meta-Analysis (V.2.0). All the tests were two-tailed and a $\mathrm{P}$ value of $<0.05$ was deemed statistically significant.

\section{Quality of evidence}

Two authors (SL, HL) independently evaluated the quality of evidence for primary and secondary outcomes according to the guidelines of the Grading of Recommendations Assessment, Development and Evaluation (GRADE) for risk of bias, inconsistency, indirectness, imprecision and publication bias. ${ }^{28}$ Each assessment result was rated as very low, low, moderate or high. Summary tables were constructed using GRADE Profiler (V.3.6).

\section{RESULTS}

\section{Search results}

A total of 1187 articles were obtained through electronic and hand searches. After 460 duplicates were removed, the titles and abstracts of 727 records were reviewed, 713 records were excluded for not meeting the inclusion criteria and thus the remaining 14 articles were retrieved, all written in English, for further assessment. Seven trials were excluded due to reports of repeated data, ${ }^{1011} 19$ not about 'combination therapy' 2930 or the control group was not monotherapy. ${ }^{25} 31$ Seven trials ${ }^{12-18}$ fulfilled our inclusion criteria and were included in our meta-analysis (figure 1).

\section{Characteristics of included trials}

The main characteristics of the included trials are summarised in table 1 . These trials were published from 2003 to 2013 and involved totally 747 patients, with the sample sizes ranging from 19 to 275 . The anabolic agents included PTH 1-34 (teriparatide) and PTH 1-84. There were two different doses (20 and 40 $\mu \mathrm{g}$ ) of PTH 1-34 included in this study. Currently, the approved dose of PTH 1-34 for the treatment of osteoporosis was $20 \mu \mathrm{g}$. The bisphosphonates included alendronate (ALN), risedronate and zoledronate. Bisphosphonates were used with a conventional dose for the treatment of osteoporosis. The duration of treatment was from 9 to 24 months. All patients received oral calcium and vitamin D supplements daily.

\section{Risk of bias assessment}

Figure 2 summarises the details of risk of bias. Only one $\operatorname{trial}^{18}$ did not clearly report the random sequence generation. Only two trials ${ }^{12}{ }^{14}$ clearly reported the allocation concealment. The treatment was not blinded to the participants in four trials. ${ }^{13}{ }^{16-18}$ Blinding of outcome assessment was adequately reported in all the included trials. ${ }^{12-18}$ There was a low risk of attrition bias, reporting bias and other biases in all trials except for one ${ }^{12}$ that had a small simple size (less than 30 ).

\section{The primary outcome: mean per cent changes in BMD of 6 to 12 months duration}

This analysis involved five ${ }^{12-141618}$ trials with a total of 615 to 627 patients. The combination therapy demonstrated a significant advantage over a monotherapy in BMD improvement at the total hip (SMD 0.42; 95\% CI 


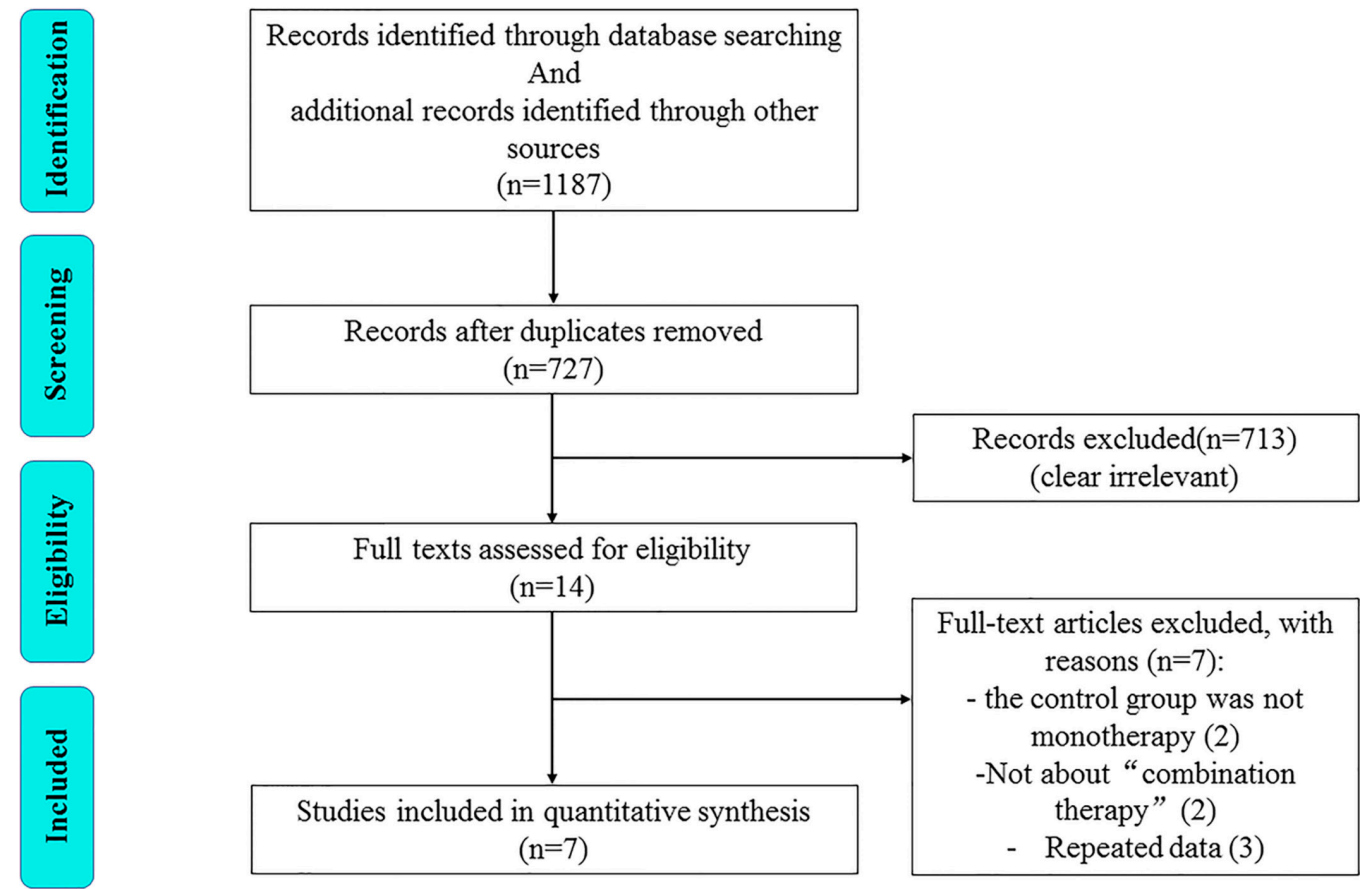

Figure 1 Flow diagram showing the process of literature selection.

0.26 to $0.58, \mathrm{I}^{2}=0 \%, \mathrm{P}<0.01$ ) and the femoral neck (SMD $0.30 ; 95 \%$ CI 0.14 to $0.46, \mathrm{I}^{2}=0 \%, \mathrm{P}<0.01$ ), although the effect of the combination therapy was equal to the monotherapy at the lumbar spine (SMD $0.13 ; 95 \%$ CI -0.17 to $0.43, \mathrm{I}^{2}=64 \%, \mathrm{P}=0.39$ ) (figure 3 ).

The sensitivity analyses showed that one trial ${ }^{16}$ significantly affected the pooled SMD at the lumbar spine, after it was omitted, and there was no significant heterogeneity (SMD $0.03 ; 95 \% \mathrm{CI}-0.23$ to $0.29, \mathrm{I}^{2}=43 \%, \mathrm{P}=0.81$ ) (see online supplementary figure $\mathrm{S} 1$ ). Then, a subgroup analysis was performed based on the different methods of combination therapy, and the results showed that among the four different methods of combination therapy, only the combination therapy with PTH 1-34 and ALN, based on two trials, was superior to the monotherapy (SMD 0.46; $95 \%$ CI 0.12 to $0.79, \mathrm{I}^{2}=16 \%, \mathrm{P}<0.01$ ) (see online supplementary figure $\mathrm{S} 2$ ).

\section{The secondary outcome: mean per cent changes in BMD of 18 to 24 months duration}

Four trials ${ }^{12}{ }^{15-17}$ with 194 to 206 patients provided the BMD data and were included in the analysis (figure 4). Compared with the anabolic agents alone, the combination therapy did not improve the BMD at the lumbar spine (SMD $-0.06 ; 95 \% \mathrm{CI}-0.71$ to $0.59, \mathrm{I}^{2}=78 \%, \mathrm{P}=0.85$ ), the total hip (SMD, $0.05 ; 95 \% \mathrm{CI}-0.71$ to $0.82 ; \mathrm{I}^{2}=84 \%$; $\mathrm{P}=0.89$ ) and the femoral neck (SMD, $-0.32 ; 95 \% \mathrm{CI}-1.15$ to $0.50 ; \mathrm{I}^{2}=86 \% ; \mathrm{P}=0.44$ ).

Subgroup analyses were performed based on the different dose of anabolic agents. Based on two trials, when a regular dose $(20 \mu \mathrm{g})$ was used in the combination therapy, the BMD significantly increased at the lumbar spine (SMD $0.53 ; 95 \%$ CI 0.14 to $0.91, \mathrm{I}^{2}=0 \%, \mathrm{P}<0.01$ ) and the total hip (SMD, 0.60; 95\% CI 0.15 to 1.06 ,
$\mathrm{I}^{2}=13 \%, \mathrm{P}=0.01$ ), although the effect of the combination therapy was equal to the monotherapy at the femoral neck (SMD, $0.30 ; 95 \% \mathrm{CI}-0.37$ to $0.98, \mathrm{I}^{2}=50 \%, \mathrm{P}=0.38$ ) (see online supplementary figure S3).

In contrast, based on two trials, compared with a monotherapy with a double dose $(40 \mu \mathrm{g})$ of PTH $1-34$, the combination therapy had no advantage, and even reduced the ability of PTH 1-34 to increase the BMD at the lumbar spine (SMD $-0.54 ; 95 \% \mathrm{CI}-0.97$ to $-0.10, \mathrm{I}^{2}=0 \%, \mathrm{P}=0.02$ ), the total hip (SMD, $-0.58 ; 95 \% \mathrm{CI}-1.31$ to $0.14, \mathrm{I}^{2}=63 \%$, $\mathrm{P}=0.12$ ) and the femoral neck (SMD, $-1.02 ; 95 \%$ CI -2.02 to $-0.02, \mathrm{I}^{2}=78 \%, \mathrm{P}=0.38$ ) (see online supplementary figure S3).

\section{Publication bias}

The publication bias of the primary outcomes was assessed through a visual inspection of the funnel plots (figure 5). The funnel plots seemed to be asymmetric, suggesting the potential risk of publication bias.

\section{Grading the quality of evidence}

GRADE evidence profiles for each outcome are shown in table 2. The quality of evidence was judged as low for all the outcomes, which was downgraded from high due to the risk of bias and the potential reporting bias.

\section{DISCUSSION}

\section{Main findings}

We performed this meta-analysis of seven RCTs, involving a total of 747 patients, to determine whether the concomitant combination therapy of anabolic agents and bisphosphonates produces more effects on BMD than anabolic agents alone in patients with osteoporosis. The results 


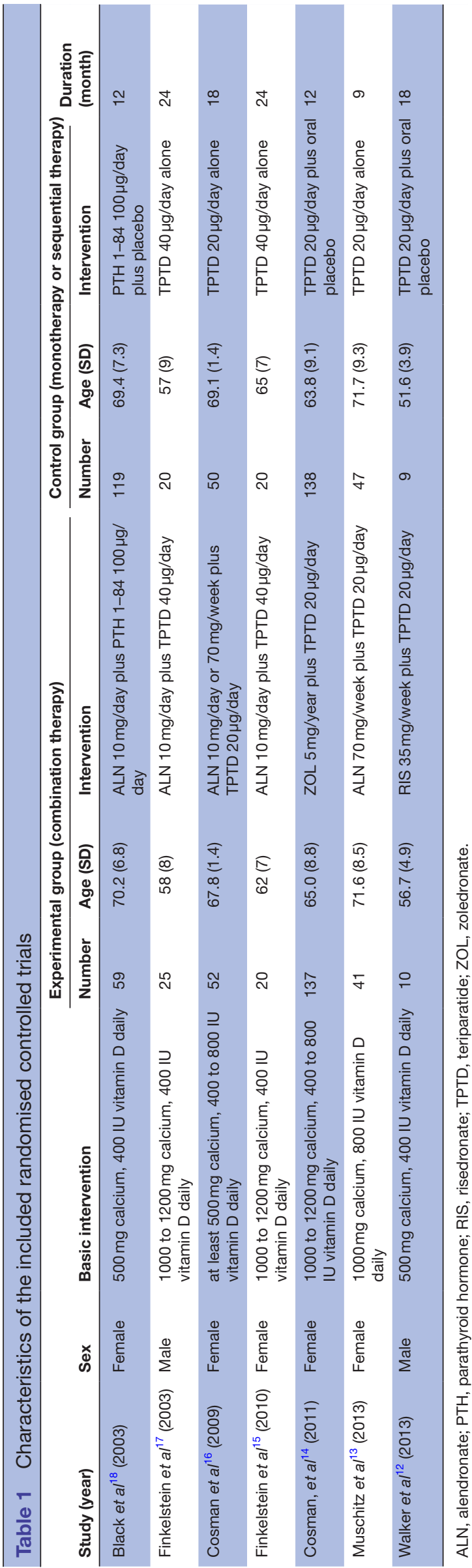

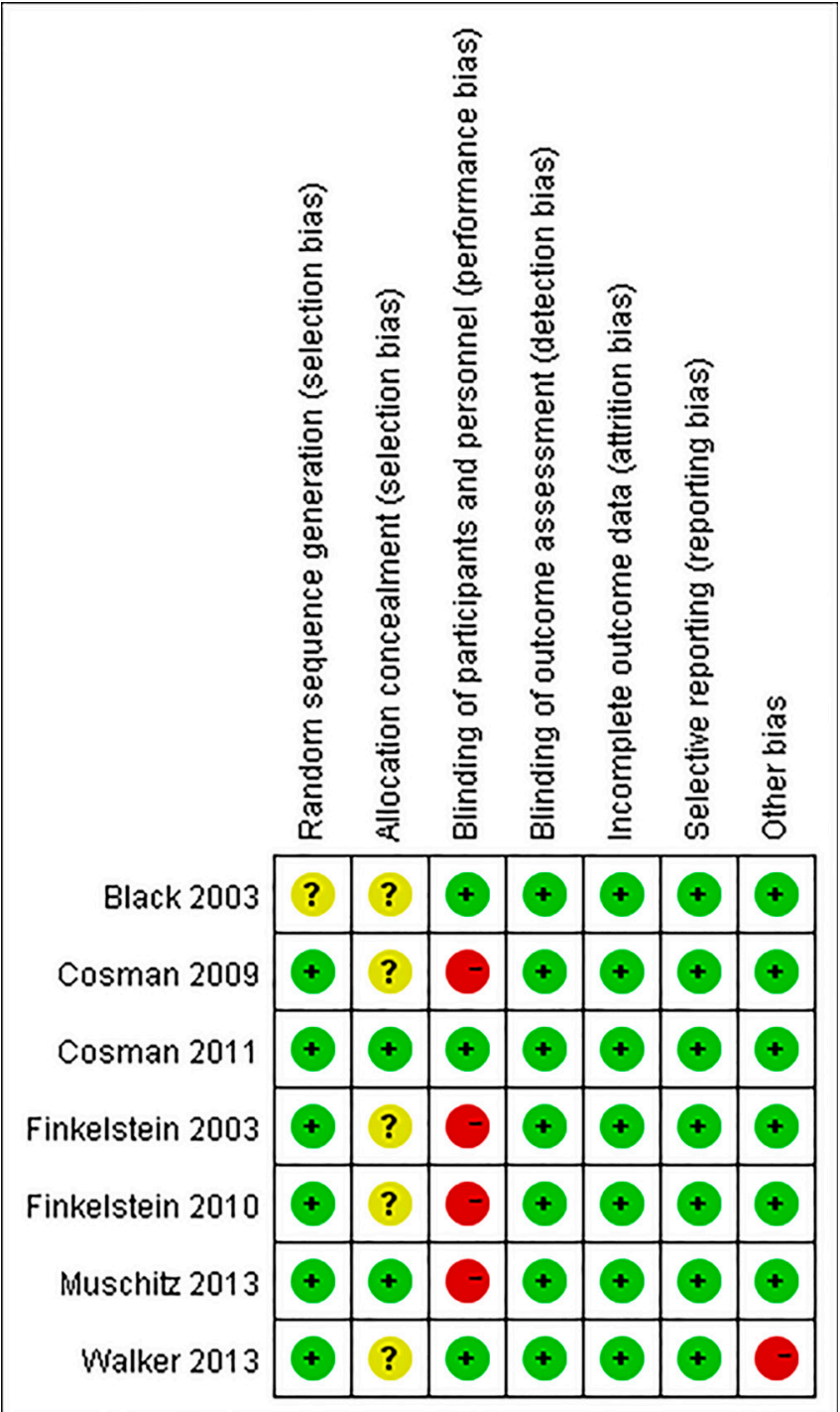

Figure 2 Risk of bias graph. Risk of bias summary. "+" means low risk; "?" means unclear risk; '-" means high risk.

of our pooled analysis indicated that compared with anabolic monotherapy, the combination therapy significantly improved the BMD at the total hip and femoral neck with a shorter term (6 to 12 months) and produced similar benefits on BMD for the longer term (18 to 24 months). In addition, subgroup analyses indicated that the effect of the combination therapy might be affected by the dose of anabolic agents.

\section{Comparison with other studies}

Our results are consistent with the previous meta-analysis by Li $e t a l$ of the fact that the combination therapy, in the first year, is superior to anabolic monotherapy for the BMD improvement at the total hip, but not for the lumbar spine BMD. ${ }^{20}$ Meanwhile, our results are compatible with Wang $e t a l^{21}$ that the dosage $(20 \mu \mathrm{g}$ or $40 \mu \mathrm{g})$ of anabolic agents could affect the effect of combination therapy. Besides that, in agreement with the previous meta-analyses,${ }^{2021}$ we also determine that, the combination therapy does not have an advantage than anabolic monotherapy 


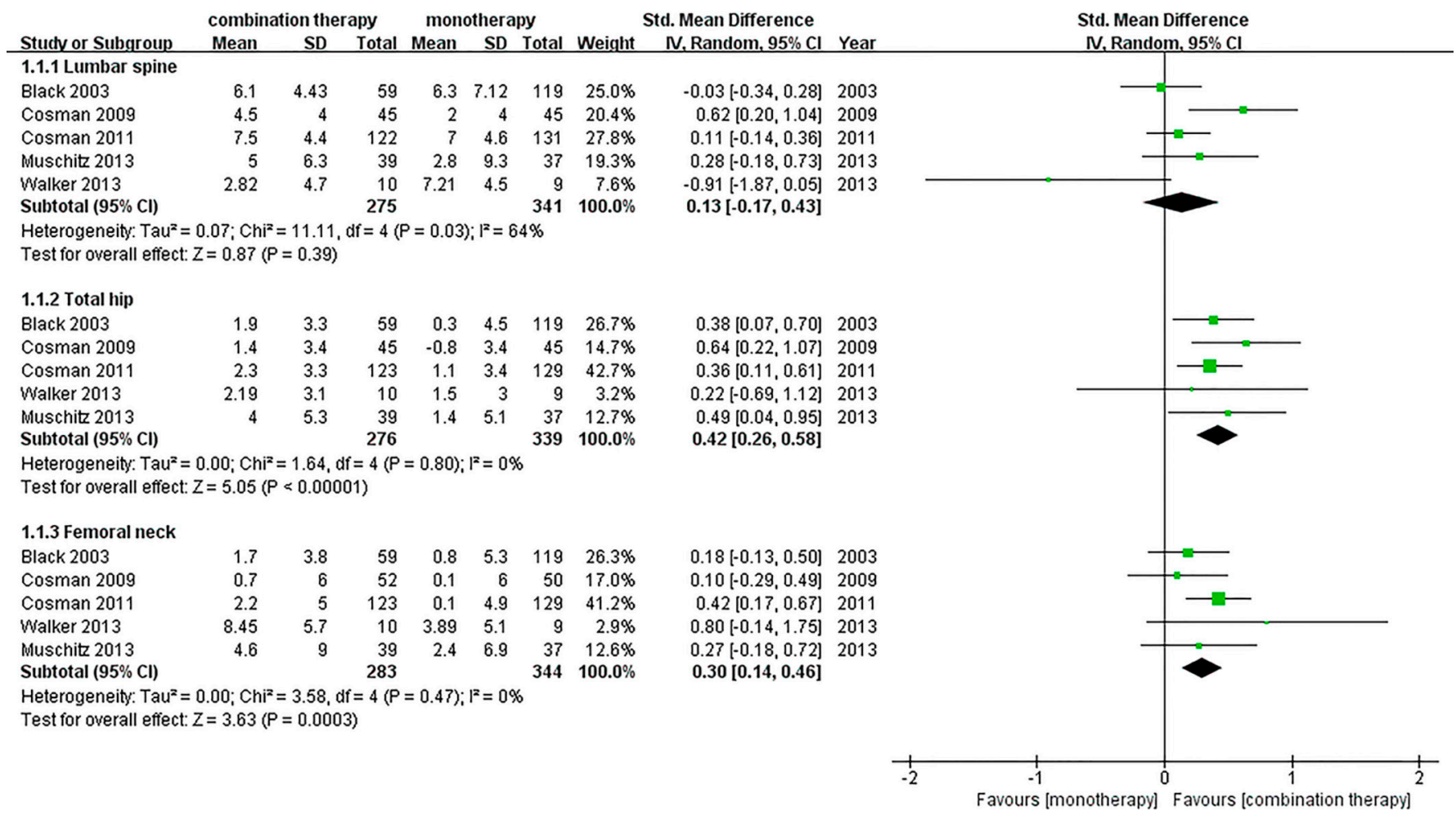

Figure 3 Forest plot for the bone mineral density variation of the 6 to 12 months duration. IV, inverse variance.

for full-length treatment (18 to 24 months). Although both the number of included trials and the pooled results between our study and the previous meta-analysis ${ }^{20} 21$ seem to be the same, it should be noted that there are some obvious differences between this study and the previous meta-analysis.

Compared with the meta-analysis by Li et $a l,{ }^{20}$ the differences are as follows: (1) Li et al only included trials with a minimum of 12-month follow-up, which led to one trial ${ }^{13}$ (included in our study) not included in their meta-analysis. Moreover, one trial ${ }^{16}$ (included in our study), which met the inclusion criteria of $\mathrm{Li} e t$ al, was missed in their study. (2) Li et al considered 'a prospective RCT comparing PTH analogues combined with bisphosphonate versus PTH analogues alone' as one of the inclusion criteria for including trials. However, the comparator of

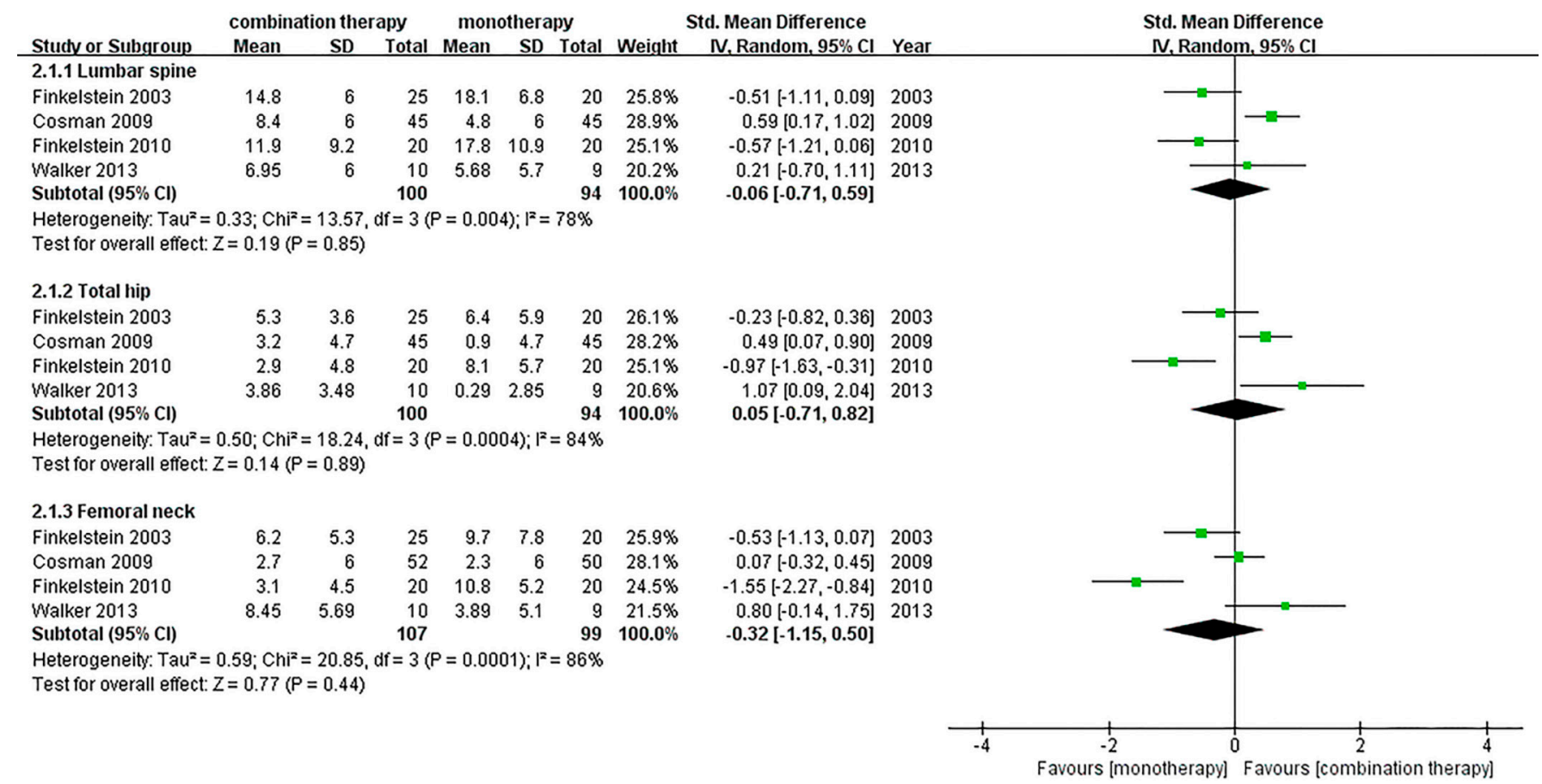

Figure 4 Forest plot for the bone mineral density variation of the 18 to 24 months duration.IV: inverse variance 


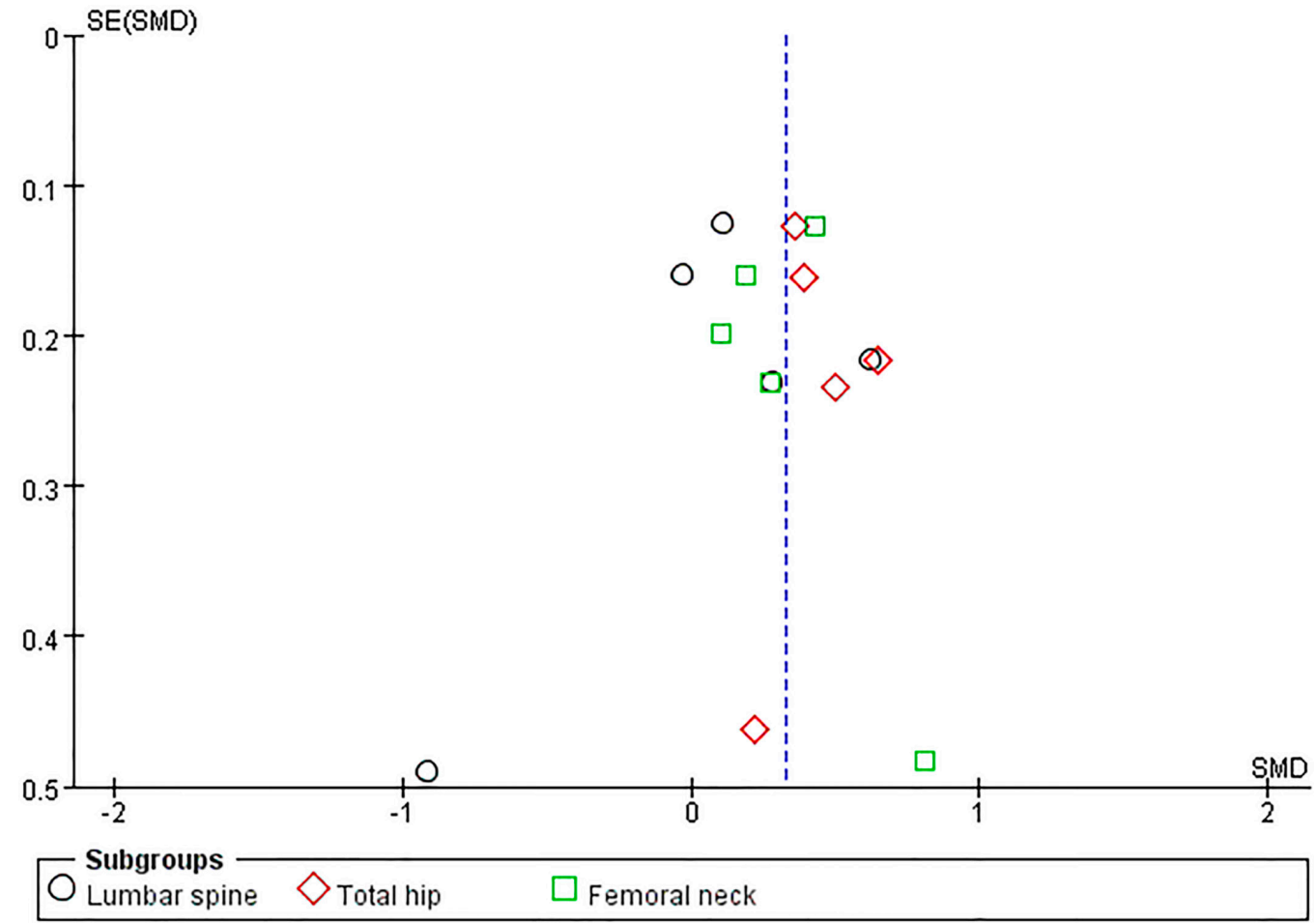

Figure 5 Funnel plot for publication bias. SMD, standardised mean difference.

one included trial ${ }^{25}$ in their meta-analysis was not 'PTH analogues alone' but 'alendronate plus parathyroid hormone (1-34) subcutaneously daily for three 3-month cycles alternating with 3-month periods without parathyroid hormone'. (3) Among the included trials of Li et $a l$, the trial Black $e t a l^{10}$ was a follow-up study of the trial Black et al, ${ }^{18}$ for the outcome 'Mean Percent Change in Hip BMD'; including both these trials led $\mathrm{Li}$ et al to use repeated data in their meta-analysis. (4) For the subgroup analyses, Li et al used different time points (12 months or 24 months) to divide the groups. But, in our meta-analysis, we used different time periods to divide the groups (6 to 12 months or 18 to 24 months), which allowed us to use more data in the meta-analysis.

Compared with the meta-analysis by Zhang $e t a l^{21}$ the differences are as follows: (1) three trials ${ }^{12-14}$ (included in our study), which meet the inclusion criteria of Zhang et $a l$, are missed in that study. (2) Zhang et al included trials should meet the inclusion criteria that "patients in the treatment group received the combined therapy of PTH with alendronate, whereas patients in the control group received other treatment'. However, the treatment group of two included trials ${ }^{32} 33$ in their meta-analysis is obviously not a combination therapy. Since one-third of the included trials (two of six) should not be included in the meta-analysis of Wang et $a l^{21}$ it may be the main reason why Wang et $a l^{21}$ made a false conclusion, in contrary to the results of our study and $\mathrm{Li} e t a l^{20}$ that 'The lumbar spine BMD with combination therapy significantly decreased at the 12-month period, but increased over the 12-month period'.
Meanwhile, owing to these differences, our results, in contrary to the study of $\mathrm{Li} e t a l,{ }^{20}$ show that the combination therapy has an advantage of increasing the femoral neck BMD than anabolic monotherapy in the first year. Furthermore, although some of our results are consistent with Li $e t a l^{20}$ and Wang $e t a l,{ }^{21}$ it should be noted that our study is more rigorous, making our results more credible than those two previous meta-analyses.

\section{Implications for clinical practice}

Since anabolic agents were approved for a limited period (18 to 24 months), a sequential therapy was required due to the short duration. According to current evidence, as a part of the sequential therapy, using the combination therapy at the second step, switching from monotherapy (anabolic or antiresorptive agents) to combination therapy, might be appropriate. ${ }^{10} 13163435$ Moreover, anabolic agents could be used concomitantly combined with other antiresorptive agents. The effects of combination therapy, also, seem to be affected by the potency of antiresorptive agents. ${ }^{36}$ The simultaneous use of teriparatide and denosumab, a more potent antiresorptive agent than bisphosphonates, was more effective than monotherapy with anabolic agents, ${ }^{37} 38$ and the BMD changes were larger than other combination strategies. ${ }^{7}$ In agreement with our study, the simultaneous use of teriparatide and denosumab was only superior to monotherapy during the first year of treatment, ${ }^{37}$ but not thereafter. ${ }^{39}$ Thus, based on the current information, maybe, the concomitant combined therapy should be limited to 1 year. 


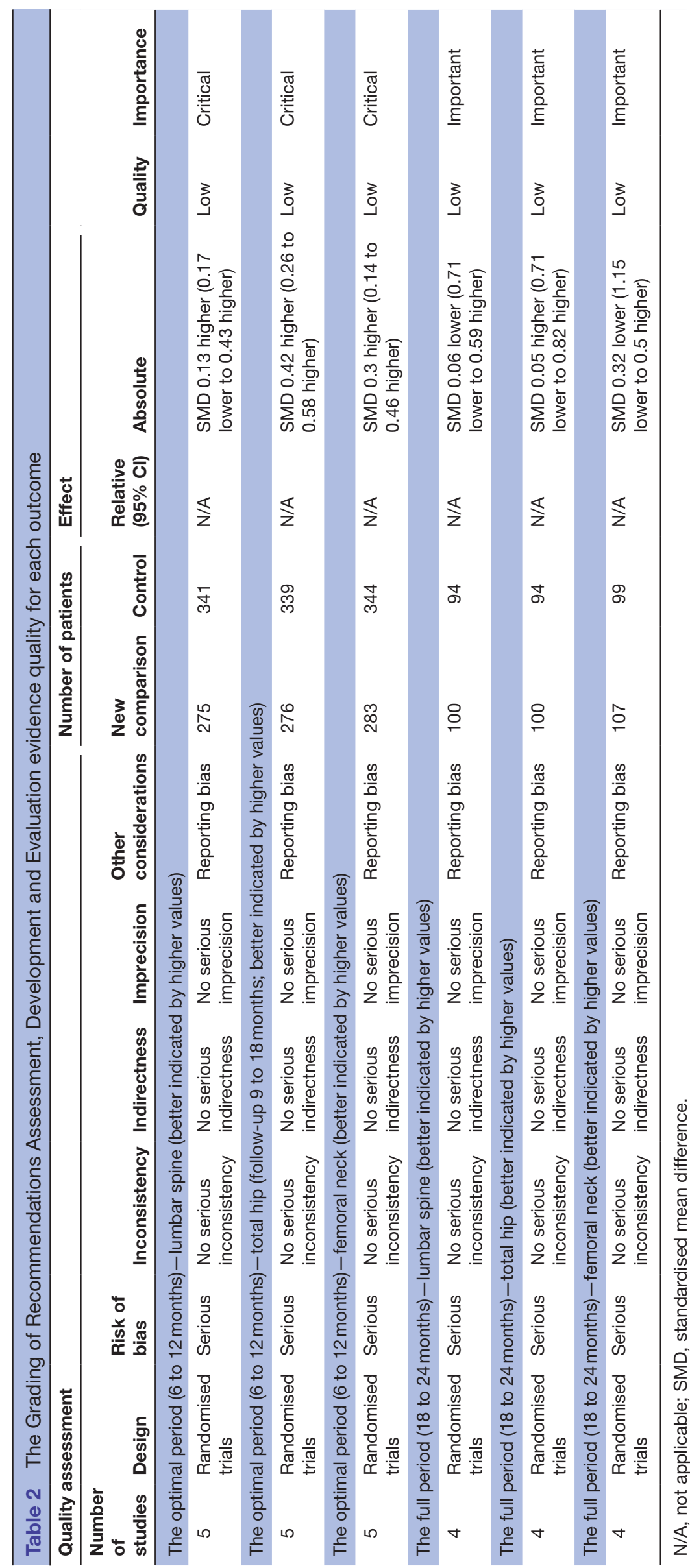

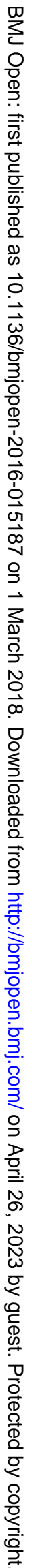


Although current evidence does not yet support a change in clinical practice, in our clinical opinion, concomitant combination therapy may be appropriate for certain people with osteoporosis, for example, for people previously treated with antiosteoporotic treatment who yet continue to lose significant bone density while receiving monotherapy, additionally, for those patients who have a high risk of fracture, who have multiple prior fractures or who continue to fracture.

\section{Limitations}

This study has limitations. (1) The number of the included studies was small, which might lead to a risk of bias in reporting the results and limit a quantitative analysis of the publication bias. (2) There were some methodological limitations in the included trials, for instance, there was an unclear random sequence generation, an unclear allocation concealment, a lack of blindness of the patients and small sample sizes. (3) Since the sample size of each trial was fairly small, a direct assessment of the antifracture efficacy was not performed. (4) Due to the small number of studies, there was limited ability to consider subgroup analyses.

\section{Implications for future research}

Further studies should focus on the following points. (1) Although BMD has been proven to be a reliable predictor of antifracture efficacy in patients treated with antiosteoporosis drugs, ${ }^{40-43}$ further studies should pay more attention to both the BMD endpoints and the fracture endpoints. (2) Owing to the small number of studies, both the shortterm (6 to 12 months) and the long-term (18 to 24 months) effect of the concomitant combination therapy still need further investigation with additional trials. (3) Although the concomitant combination therapy showed no benefit for the long-term (18 to 24 months), our subgroup analyses suggest that the teriparatide of $20 \mu \mathrm{g}$ as combination therapy provided an advantage over the anabolic monotherapy over this long term. Further trials are needed to verify this outcome, and determine whether the effect of concomitant combination therapy is to be affected by the dose and type of anabolic agents. (4) Since the use of combination therapy carries a potential risk of more adverse events, future studies should investigate both the short-term and long-term adverse events of combination therapy. (5) To date, a variety of bisphosphonates were approved for the treatment of osteoporosis, which was more appropriate using in combination therapy is unclear. (6) Among the seven included trials, most patients included in this meta-analysis were osteoporotic women. Since the results were mainly carried out in women, whether the results presented in this meta-analysis are applicable to osteoporotic men need to be further studied.

\section{CONCLUSIONS}

Among patients with osteoporosis, compared with anabolic monotherapy, the concomitant combination therapy of anabolic agents and bisphosphonates significantly improved the BMD at the total hip and femoral neck with a shorter term (6 to 12 months) and produced similar benefits on BMD for the longer term (18 to 24 months). Also, the effect of concomitant combination therapy might be affected by the dose of anabolic agents.

Contributors PT is the guarantor. PT, LZ and SL contributed to the conception and design of this study protocol. SL registered the protocol with the PROSPERO database. The search strategy was developed by SL and ZL. SL and HL selected the studies for inclusion and extracted the data. ZL, SL and HL did the analyses. LZ and PT gave advice on the data analysis and the presentation of the study results. All the authors drafted and critically reviewed this manuscript and approved the final version.

Funding This research received no specific grant from any funding agency in the public, commercial or not-for-profit sectors.

Competing interests None declared.

Patient consent Not required.

Provenance and peer review Not commissioned; externally peer reviewed.

Data sharing statement No additional data are available.

Open Access This is an Open Access article distributed in accordance with the Creative Commons Attribution Non Commercial (CC BY-NC 4.0) license, which permits others to distribute, remix, adapt, build upon this work non-commercially, and license their derivative works on different terms, provided the original work is properly cited and the use is non-commercial. See: http://creativecommons.org/ licenses/by-nc/4.0/

(c) Article author(s) (or their employer(s) unless otherwise stated in the text of the article) 2018. All rights reserved. No commercial use is permitted unless otherwise expressly granted.

\section{REFERENCES}

1. NIH Consensus Development Panel on Osteoporosis Prevention, Diagnosis, and Therapy. Osteoporosis prevention, diagnosis, and therapy. JAMA 2001;285:785-95.

2. Cummings SR, Melton LJ. Epidemiology and outcomes of osteoporotic fractures. The Lancet 2002;359:1761-7.

3. Johnell O, Kanis JA. An estimate of the worldwide prevalence, mortality and disability associated with hip fracture. Osteoporos Int 2004;15:897-902.

4. Johnell O, Kanis JA. An estimate of the worldwide prevalence and disability associated with osteoporotic fractures. Osteoporos Int 2006;17:1726-33.

5. Burge R, Dawson-Hughes B, Solomon DH, et al. Incidence and economic burden of osteoporosis-related fractures in the United States, 2005-2025. J Bone Miner Res 2007;22:465-75.

6. Cosman F. Combination therapy for osteoporosis: a reappraisal. Bonekey Rep 2014;3:518.

7. McClung MR. Using osteoporosis therapies in combination. Curr Osteoporos Rep 2017;15:343-52.

8. Bilezikian JP. Combination anabolic and antiresorptive therapy for osteoporosis: opening the anabolic window. Curr Osteoporos Rep 2008;6:24-30.

9. Chen JS, Sambrook PN. Antiresorptive therapies for osteoporosis: a clinical overview. Nat Rev Endocrinol 2011;8:81-91.

10. Black DM, Bilezikian JP, Ensrud KE, et al. One year of alendronate after one year of parathyroid hormone (1-84) for osteoporosis. $N$ Engl J Med 2005;353:555-65.

11. Finkelstein JS, Leder BZ, Burnett SM, et al. Effects of teriparatide, alendronate, or both on bone turnover in osteoporotic men. $J$ Clin Endocrinol Metab 2006;91:2882-7.

12. Walker MD, Cusano NE, Sliney J, et al. Combination therapy with risedronate and teriparatide in male osteoporosis. Endocrine 2013;44:237-46.

13. Muschitz C, Kocijan R, Fahrleitner-Pammer A, et al. Antiresorptives overlapping ongoing teriparatide treatment result in additional increases in bone mineral density. J Bone Miner Res 2013;28:196-205.

14. Cosman F, Eriksen EF, Recknor C, et al. Effects of intravenous zoledronic acid plus subcutaneous teriparatide [rhPTH(1-34)] in postmenopausal osteoporosis. J Bone Miner Res 2011;26:503-11.

15. Finkelstein JS, Wyland JJ, Lee H, et al. Effects of teriparatide, alendronate, or both in women with postmenopausal osteoporosis. $J$ Clin Endocrinol Metab 2010;95:1838-45. 
16. Cosman F, Wermers RA, Recknor C, et al. Effects of teriparatide in postmenopausal women with osteoporosis on prior alendronate or raloxifene: differences between stopping and continuing the antiresorptive agent. J Clin Endocrinol Metab 2009;94:3772-80.

17. Finkelstein JS, Hayes A, Hunzelman JL, et al. The effects of parathyroid hormone, alendronate, or both in men with osteoporosis. N Engl J Med 2003;349:1216-26.

18. Black DM, Greenspan SL, Ensrud KE, et al. The effects of parathyroid hormone and alendronate alone or in combination in postmenopausal osteoporosis. N Engl J Med 2003;349:1207-15.

19. Wermers RA, Recknor CP, Cosman F, et al. Effects of teriparatide on serum calcium in postmenopausal women with osteoporosis previously treated with raloxifene or alendronate. Osteoporos Int 2008;19:1055-65.

20. Li W, Chen W, Lin Y. The efficacy of parathyroid hormone analogues in combination with bisphosphonates for the treatment of osteoporosis: a meta-analysis of randomized controlled trials. Medicine 2015;94:e1156.

21. Wang C, Zhang G, Gu M, et al. Parathyroid hormone plus alendronate in osteoporosis: a meta-analysis of randomized controlled trials. J Invest Surg 2015;28:309-16.

22. Moher D, Liberati A, Tetzlaff J, et al. Preferred reporting items for systematic reviews and meta-analyses: the PRISMA statement. PLoS Med 2009;6:e1000097.

23. Whitaker M, Guo J, Kehoe T, et al. Bisphosphonates for osteoporosis--where do we go from here? N Engl J Med 2012;366:2048-51.

24. Black DM, Bauer DC, Schwartz AV, et al. Continuing bisphosphonate treatment for osteoporosis--for whom and for how long? N Engl J Med 2012;366:2051-3.

25. Cosman F, Nieves J, Zion M, et al. Daily and cyclic parathyroid hormone in women receiving alendronate. $N$ Engl $J$ Med 2005;353:566-75.

26. Higgins JP, Altman DG, Gøtzsche PC, et al. The Cochrane Collaboration's tool for assessing risk of bias in randomised trials. BMJ 2011;343:d5928.

27. Higgins JP, Thompson SG, Deeks JJ, et al. Measuring inconsistency in meta-analyses. BMJ 2003;327:557-60.

28. Atkins D, Best D, Briss PA, et al. Grading quality of evidence and strength of recommendations. BMJ 2004;328:1490.

29. Finkelstein JS, Wyland JJ, Leder BZ, et al. Effects of teriparatide retreatment in osteoporotic men and women. $J$ Clin Endocrinol Metab 2009;94:2495-501.

30. Cosman F, Nieves JW, Zion M, et al. Retreatment with teriparatide one year after the first teriparatide course in patients on continued long-term alendronate. J Bone Miner Res 2009;24:1110-5.
31. Schafer AL, Sellmeyer DE, Palermo L, et al. Six months of parathyroid Hormone (1-84) administered concurrently versus sequentially with monthly ibandronate over two years: the PTH and ibandronate combination study (PICS) randomized trial. J Clin Endocrinol Metab 2012;97:3522-9.

32. Boonen S, Marin F, Obermayer-Pietsch B, et al. Effects of previous antiresorptive therapy on the bone mineral density response to two years of teriparatide treatment in postmenopausal women with osteoporosis. J Clin Endocrinol Metab 2008;93:852-60.

33. Miller PD, Delmas PD, Lindsay R, et al. Early responsiveness of women with osteoporosis to teriparatide after therapy with alendronate or risedronate. J Clin Endocrinol Metab 2008:93:3785-93.

34. Lou S, Lv H, Wang G, et al. The effect of sequential therapy for postmenopausal women with osteoporosis: A PRISMAcompliant meta-analysis of randomized controlled trials. Medicine 2016;95:e5496.

35. Leder BZ, Tsai JN, Uihlein AV, et al. Denosumab and teriparatide transitions in postmenopausal osteoporosis (the DATA-Switch study): extension of a randomised controlled trial. Lancet 2015;386:1147-55.

36. Shen Y, Gray DL, Martinez DS. Combined pharmacologic therapy in postmenopausal osteoporosis. Endocrinol Metab Clin North Am 2017;46:193-206.

37. Tsai JN, Uihlein AV, Lee H, et al. Teriparatide and denosumab, alone or combined, in women with postmenopausal osteoporosis: the DATA study randomised trial. Lancet 2013;382:50-6.

38. Idolazzi L, Rossini M, Viapiana O, et al. Teriparatide and denosumab combination therapy and skeletal metabolism. Osteoporos Int 2016;27:3301-7.

39. Leder BZ, Tsai JN, Uihlein AV, et al. Two years of Denosumab and teriparatide administration in postmenopausal women with osteoporosis (The DATA Extension Study): a randomized controlled trial. J Clin Endocrinol Metab 2014;99:1694-700.

40. Hochberg MC, Greenspan S, Wasnich RD, et al. Changes in bone density and turnover explain the reductions in incidence of nonvertebral fractures that occur during treatment with antiresorptive agents. J Clin Endocrinol Metab 2002;87:1586-92.

41. Cummings SR, Karpf DB, Harris F, et al. Improvement in spine bone density and reduction in risk of vertebral fractures during treatment with antiresorptive drugs. Am J Med 2002;112:281-9.

42. Chen P, Miller PD, Delmas PD, et al. Change in lumbar spine $\mathrm{BMD}$ and vertebral fracture risk reduction in teriparatide-treated postmenopausal women with osteoporosis. J Bone Miner Res 2006;21:1785-90.

43. Khosla S. Surrogates for fracture endpoints in clinical trials. J Bone Miner Res 2003;18:1146-9. 